\title{
Active joints for microrobot limbs
}

\section{$M$ Elwenspoekt, L Smith and B Hök}

Department of Technology, Uppsala University, PO Box 534, S-751 21 Uppsala, Sweden

\begin{abstract}
In this contribution we propose an electrostatic actuator for active joints. The active joint consists of two plates, one of which is a bilayer and bent by the bimorph effect. The plates are clamped to each other at one edge. A voltage between the plates leads to a very large field at the clamp where the plates are in intimate contact, thereby producing a force large enough to pull the bent bimorph cantilever beam to the other plate. In this design we have an actuator in which large electrical forces acting over a short distance are used to produce large deflections. We see an appealing application of this actuation principle in active joints for robot arms, e.g. by attaching members to the actuator and by combining two or more active joints and members to form micro robot arms, legs and grippers.
\end{abstract}

A great challenge for microtechnology is the development of microrobot systems. An impressive discussion of the potential of microrobots can be found in [1]. Armadas of autonomous insect-sized microrobots could be sent in tiny tube systems, motors or even into the human body to perform inspection and repair.

This is of course still science fiction. There is no suitable means for power supply, artificial brains capable of autonomous navigation are still much too massive, and the actuators developed so far do not seem to be useful for e.g. the locomotion drive.

In this contribution we propose the use of active joints for limbs in microrobots. In an active joint, the actuation that serves for imposing a controllable angle between stiff members and the joint that connects them are integrated in a single component.

The idea of active joints is based on recent work of Branebjerg and Gravesen [2], which is closely related to work of Sato and Shikida [3] and Ohnstein et al [4]. The actuator consists of two plates, at least one of which is deformable. A voltage between the plates produces an attractive force, and the plates come close to each other. At the clamping point the distance between the plates must be very small, in the range of a few tenths of a $\mu \mathrm{m}$, and the force will be very large there. If it is large enough it will pull down the whole deformable plate which results in large forces combined with a large displacement. The counterforce, which bends the movable plate away from the fixed plate, has been provided in earlier work by a pressure, the deformable plate being a membrane [2], by electrostatic force [3], and by the pressure drop of a streaming fluid [4].

Here we propose a construction where the counterforce is provided by elastic forces of a bimorph. This principal idea is illustrated in figure 1.

tOn sabbatical leave from MESA-Research Institute, University of Twente, NL-7550 AE Enschede, The Netherlands.
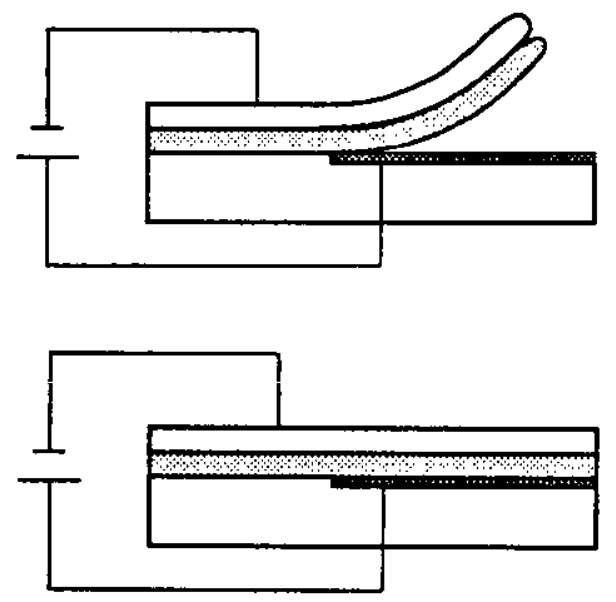

Figure 1. Schematic of the active joint. The bilayer on top of the structure is bonded to the left of the bottom electrode and free above the electrode. Top: unactivated, bottom: fully activated.

To get an idea of the performance of this actuator we made a model in which we only considered the electrostatic field of that part of the structure which is collapsed, i.e. where the electrodes are the shortest possible distance apart. The total electrostatic energy $E_{\mathrm{es}}$ is given by

$$
E_{\text {es }}=\frac{1}{2} \frac{\varepsilon \varepsilon_{0}}{d} A V^{2}
$$

where $\varepsilon$ and $\varepsilon_{0}$ are relative and absolute dielectric constants, $d$ the distance between the electrodes, $A$ their area in contact, and $V$ is the voltage between the electrodes. For rectangular capacitor plates, $A=b^{\prime} l$, with $b^{\prime}$ the width of the capacitor plate.

If a bent cantilever beam is flattened, the work to achieve this is given by

$$
E_{\mathrm{e} 1}=-\frac{E h^{3} b l}{24 R^{2}}
$$


where $E$ is the effective Youngs' modulus of the bimorph, $h$ its thickness, $b$ the width, $l$ the length over which the beam has been straightened, and $R$ the radius of curvature of the bent bimorph. There is equilibrium if

$$
\frac{\partial E}{\partial l}=\frac{\partial}{\partial l}\left(E_{\mathrm{es}}+E_{\mathrm{el}}\right)=0
$$

and it is easily seen that for rectangular capacitor plates there is no equilibrium, since the elastic and the electrostatic energy have the same dependence on $l$. Therefore the structure is a two-state system: either it will be activated, the flexible plate lying flat on the fixed plate, or it is inactivated, the whole bimorph being bent away from the fixed plate. This character applies for the devices described in [2-4] as well. The voltage at which the turnover point occurs is found from $E_{\mathrm{el}}+E_{\mathrm{es}}=0$, from which we get

$$
V_{\mathrm{c}}=\sqrt{\frac{E h^{3} d}{12 R^{2} \varepsilon \varepsilon_{0}}}
$$

where we have set $b=b^{\prime}$. For $E=10^{11} \mathrm{~N} \mathrm{~m}^{-2}$, $d=h=1 \mu \mathrm{m}, R=1 \mathrm{~mm}$ and $\varepsilon=1$, we find a reasonable value of $V_{c}=30 \mathrm{~V}$.

A joint with only two stable states is of limited interest, but the discrete character can be easily removed if one makes use of appropriately shaped electrodes. As an example consider a triangular shaped electrode with base $b_{0}$ and length $L$. Now $A$, the surface of the contacted part of the electrode depends quadratically on $l$,

$$
A=b_{0} l\left[1-\frac{l}{2 L}\right]
$$

and equation (3) leads now to a minimum in the total energy at

$$
l=L\left\{1-\left[\frac{V_{\mathrm{c}}}{V}\right]^{2}\right\}
$$

where $V_{\mathrm{c}}$ is given by (4). $l$ is the length of the contacted electrodes and in contrast to the actuators discussed so far $l$ depends on the voltage. The deflection of the actuator is now controllable. Above $V_{c}, l$ becomes larger than 0 , and equal to $L$ for infinitely large voltages. The steep dependence of $l$ on the voltage leads to a reasonable deflection at reasonable voltages. As an example, if $V_{\mathrm{c}}=30 \mathrm{~V}, 90 \%$ of the actuator will be collapsed at a voltage of $100 \mathrm{~V}$.

The radius of curvature depends on the materials used, the thickness of the layers and the production process, e.g. a bimorph of $1 \mu \mathrm{m}$ of each $\mathrm{Si}$ and $\mathrm{SiO}_{2}$ can have a radius of curvature of the order of $1 \mathrm{~mm}$; use of $\mathrm{Al}$ could reduce $R$ further and polymers, having a volume shrinkage of nearly $10 \%$, would lead to even smaller values of $R$.

There are many schemes for the realization of such a device. In [2] a combination of anodic bonding and bulk micromachining is used to fabricate the structure, and in [4] surface micromachining is used, with silicon nitride and an encapsulated metal electrode for the movable plate. Thus the fabrication of the basic structure should pose no difficulties at all. A challenge would be the introduction of new materials such as PZT $(\varepsilon=1300$ [1]) as a dielectricum.

As has been pointed out by Branebjerg and Gravesen [2], the actuator only functions properly if the plates are in contact at the edge from the very beginning. The sacrificial layer therefore must be very thin, of the order of $100 \mathrm{~nm}$, or there has to be a static moment in the movable plate that bends the plate towards the fixed plate. This requirement poses some complication on the fábrication process, bùt no fundamentâl difficultiess.

There is a serious drawback of the devices, that has also been mentioned by Branebjerg and Gravesen [2]. Contamination by dust between the plates will prevent the plates bending to make intimate contact, and will therefore be fatal for the actuator. This limits the actuator either to application in specific environments only (high vacuum or clean room environments) or to a rather limited lifetime. This drawback motivated Branebjerg and Gravesen to use a membrane instead of a cantilever beam, since a membrane can be sealed hermetically [2].

We conclude with brief descriptions of two examples for applications in microrobots.

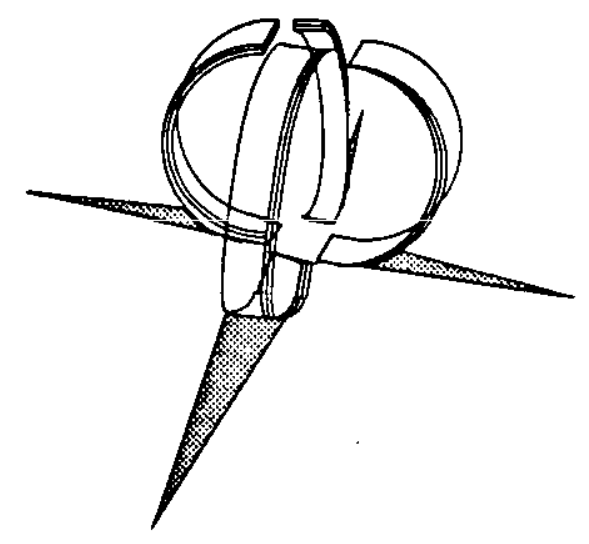

(a)

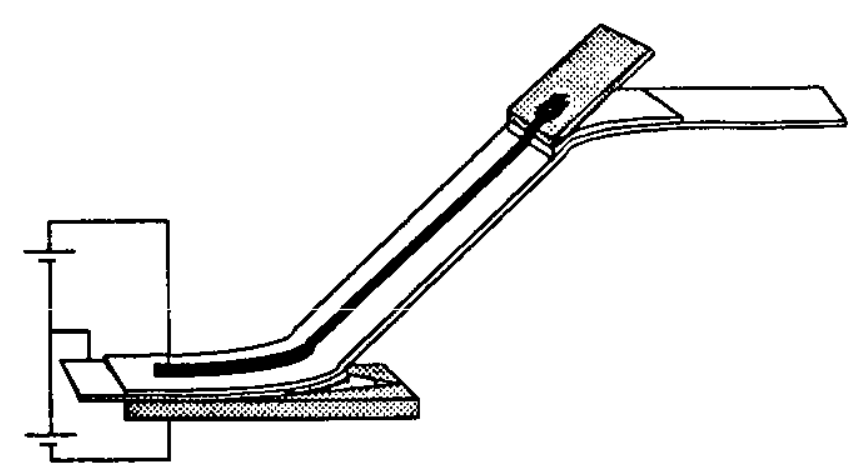

(b)

Figure 2. (a) Schematic of a micro hand using a combination of active joints. Note the triangular shaped electrodes. (b) Schematic of a micro robot arm with two active joints and members. 
(1) Several active joints can be combined to form grippers simply by e.g. two plates that bend by $180^{\circ}$ oriented opposite to each other. Even the realization of a hand could be possible. An illustration is given in figure $2(a)$.

(2) The realization of a robot arm with two or more active joints should be possible. The joints could be oriented in a common direction as in human fingers, or in opposite directions as in insect legs. An illustration is given in figure $2(b)$.

The realization of a well performing active joint for microrobot applications such as arms, legs and grippers based on electrostatic and elastic forces seems to be feasible. Using well established materials active joints can be fabricated having radii of curvature of less than $1 \mathrm{~mm}$, with a thickness of the order of $1 \mu \mathrm{m}$ using straightforward production schemes. Improvement aiming at thicker and stronger structures requires the introduction of less well established materials such as PZT. The introduction of active joints could provide a step closer to the development of autonomous microrobots. More down to earth applications could be found in micro adjustment systems, micro liquid handling systems (pumps and valves) and micro manipulators.

\section{Acknowledgments}

One of us (ME) wishes to thank the Uppsala micromechanics group for their hospitality. Helpful discussions with Jan Söderkwist are gratefully acknowledged.

\section{References}

[1] Flynn A M, Tavrow L S, Bart S F, Brooks R A, Ehrlich D J, Udayakumar $\mathrm{K} R$ and Cross $\bar{L}$ E $19 \overline{9} 2$ Piezoelectric micromotors for microrobots $J$ Microelectromech. Systems 144

[2] Branebjerg J and Gravesen P 1992 A new electrostatic actuator providing improved stroke length and force Proc. Micro Electro Mechanical Systems (Travemunde, Germany, 1992) p 6

[3] Sato K and Shikida M 1992 Electrostatic film actuator with large vertical displacement Proc. Micro Electro Mechanical Systems (Travemunde, Germany, 1992) pl

[4] Ohnstein T, Fukiura T, Ridley J and Bonne U 1990 Micromachined silicon microvalve Proc. Micro Electro Mechanical Systems (Napa Valley, CA, 1990) p 95 